\title{
Structural-Thermal-Optical-Performance (STOP) Model Development and Analysis of a Field-widened Michelson Interferometer
}

\author{
Salvatore J. Scola, James F. Osmundsen, Luke S. Murchison, Warren T. Davis, Joshua M. Fody, \\ Charles M. Boyer, Anthony L. Cook, Chris A. Hostetler, Shane T. Seaman \\ NASA Langley Research Center, 100 NASA Drive, Hampton, VA, USA 23681
}

\author{
Ian J. Miller \\ LightMachinery, 80 Colonnade Rd, Nepean, Ontario, Canada K2E 7L2 \\ Wayne C. Welch, Adam R. Kosmer \\ Welch Mechanical Designs, 1371 Brass Mill Road, Suite B, Belcamp, MD, USA 21017
}

\begin{abstract}
An integrated Structural-Thermal-Optical-Performance (STOP) model was developed for a field-widened Michelson interferometer which is being built and tested for the High Spectral Resolution Lidar (HSRL) project at NASA Langley Research Center (LaRC). The performance of the interferometer is highly sensitive to thermal expansion, changes in refractive index with temperature, temperature gradients, and deformation due to mounting stresses. Hand calculations can only predict system performance for uniform temperature changes, under the assumption that coefficient of thermal expansion (CTE) mismatch effects are negligible. An integrated STOP model was developed to investigate the effects of design modifications on the performance of the interferometer in detail, including CTE mismatch, and other threedimensional effects. The model will be used to improve the design for a future spaceflight version of the interferometer. The STOP model was developed using the Comet SimApp ${ }^{\mathrm{TM}}$ Authoring Workspace which performs automated integration between Pro-Engineer ${ }^{\circledR}$, Thermal Desktop ${ }^{\circledR}$, MSC Nastran ${ }^{\mathrm{TM}}$, SigFit ${ }^{\mathrm{TM}}$, Code VTM, and MATLAB ${ }^{\circledR}$. This is the first flight project for which LaRC has utilized Comet, and it allows a larger trade space to be studied in a shorter time than would be possible in a traditional STOP analysis. This paper describes the development of the STOP model, presents a comparison of STOP results for simple cases with hand calculations, and presents results of the correlation effort to bench-top testing of the interferometer. A trade study conducted with the STOP model which demonstrates a few simple design changes that can improve the performance seen in the lab is also presented.
\end{abstract}

Keywords: STOP, Structural, Thermal, Optical, Performance, Michelson, Interferometer, Comet, HSRL

\section{INTRODUCTION}

Since 2006, NASA Langley Research Center (LaRC) has been routinely flying high spectral resolution lidars (HSRLs) on aircraft to measure aerosols and clouds (Hair et al, 2008 ${ }^{1}$ : Burton et al., 2012 ${ }^{2}$ ). The second-generation airborne instrument, HSRL-2, implements the HSRL technique (Shipley and Eloranta, 1983 ${ }^{3}$ ) at 355 and $532 \mathrm{~nm}$, the standard backscatter technique at $1064 \mathrm{~nm}$, and is polarization sensitive at both wavelengths. Key to the HSRL technique is the spectral separation of aerosol and molecular backscatter in the lidar receiver. On HSRL-2, this spectral separation is implemented at $532 \mathrm{~nm}$ using an iodine vapor filter (Pironen and Eloranta, 1984 ${ }^{4}$; Hair et al, 2008 ${ }^{1}$ ). At $355 \mathrm{~nm}$, spectral separation is accomplished with an interferometer.

The HSRL-2 interferometer is a field-widened Michelson consisting of a glass beam splitter, a glass "solid arm", and an "air arm". A simple schematic of the concept is shown in Figure 1. The performance of the interferometer is highly sensitive to changes in temperature, which affect the index of refraction of the glass and the thermal expansion of the entire system. To achieve the best performance, the interferometer should be maintained at a constant, uniform temperature during field missions; however, this is difficult to achieve due to the changing environment in the aircraft. 


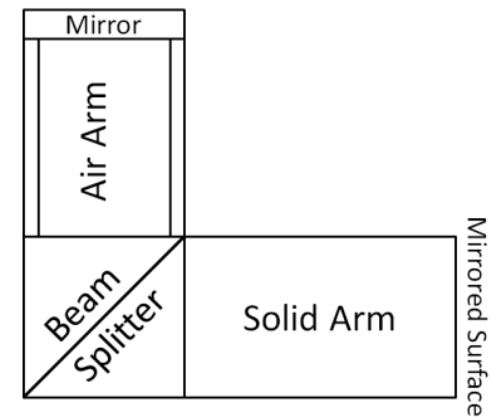

Figure 1. Interferometer schematic.

Hand calculations can be used to predict the performance of the system for uniform temperature changes under the assumption that coefficient of thermal expansion (CTE) mismatch effects are negligible. This provides a reasonable estimation of the expected system performance, but does not allow for detailed comparison of design parameters, including air arm material, component geometry, mounting configurations, and effects of non-uniform temperatures.

In order to address the limitations described above, a detailed structural-thermal-optical-performance (STOP) model was developed using the Comet SimApp ${ }^{\mathrm{TM}}$ Authoring Workspace ${ }^{5}$. Comet provides the key benefit of automating the linkage between the CAD model (physical design), and the analysis process necessary to determine the performance of the interferometer when subjected to realistic boundary conditions. By providing this linkage, geometric trade studies can be performed rapidly by updating the CAD model, and re-running the analysis process.

The purpose of this paper is to describe the development of the interferometer STOP model, and demonstrate the advantages of using a CAD-based, automated tool to perform complex, integrated analyses. A general description of the interferometer is presented, including a description of the key performance metrics for the HSRL mission. The STOP model development using Comet is described in detail, along with verification of the results with hand calculations. The results of the STOP model comparison to lab testing of the interferometer is presented, followed by a description of trade study results that will be used to improve the next iteration of the interferometer.

\section{INTERFEROMETER OVERVIEW AND PERFORMANCE METRICS}

A schematic depicting the optical function of the interferometer is shown in Figure 2. During aircraft operation of the HSRL instrument, the $355 \mathrm{~nm}$ laser is directed toward the ground, and the backscattered light from air molecules and aerosols is collected by the instrument's telescope. The collected light is collimated before entering the interferometer at a small, off-normal angle to the beam splitter face. This small angle allows both output paths of the interferometer to be collected on two separate detectors, as shown in Figure 2. The backscatter from aerosols (via Mie scattering) has a very narrow bandwidth, and if the interferometer is tuned correctly, this component of the return signal will only be present in one output of the interferometer. The molecular component of the backscatter (via Rayleigh scattering) has a bandwidth comparable to the free spectral range of the interferometer and is equally divided between the two outputs. The Mie component of the signal is the difference between the two outputs of the interferometer. Key to this technique is maintaining the optical path difference between the two arms of the Michelson so that the Mie component is only detected on one output. A single pixel detector integrates the signal for each channel over time buckets corresponding to the range resolution (altitude). 


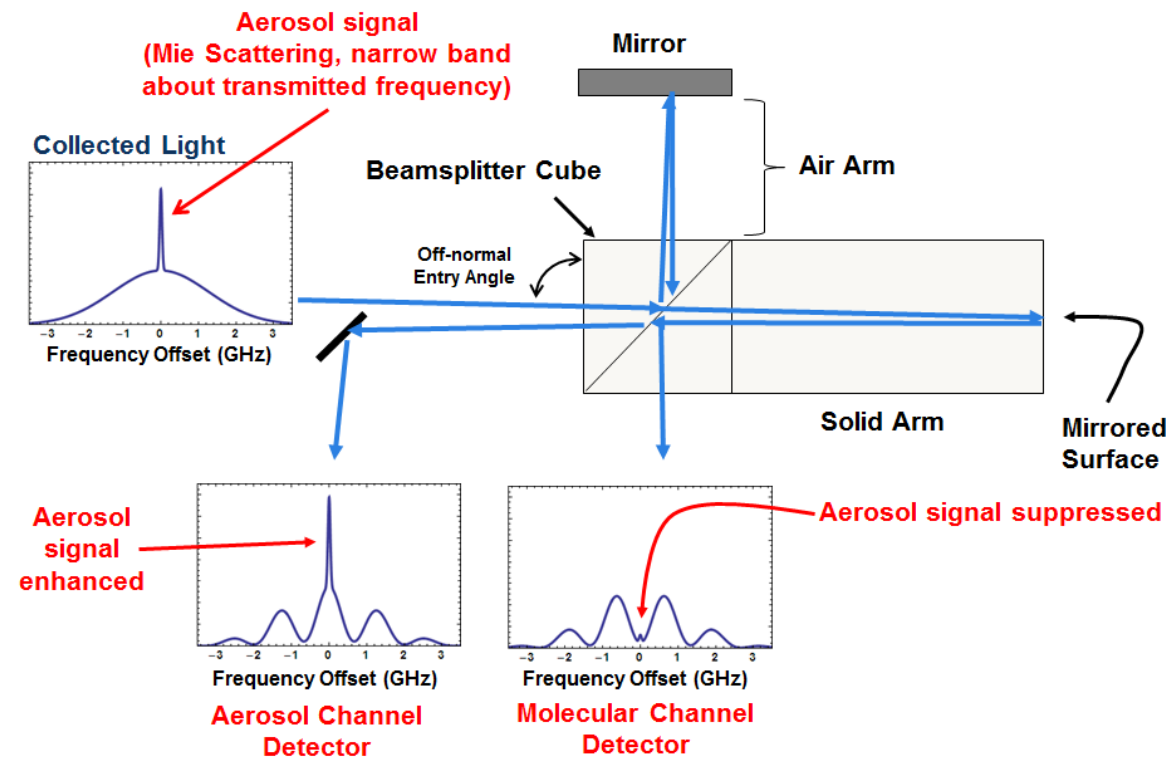

Figure 2. Operational concept of interferometer as filter.

The optical path difference (OPD) of the interferometer can held constant by changing the air pressure (air density), and thus the refractive index in the air arm. This "pressure-tuning" ability allows for compensation of changes in optical path lengths due to thermal expansion and glass refractive index changes with changes in system temperature.

A performance metric called the Aerosol Transmission Ratio (ATR) is defined in order to measure how well the interferometer is suppressing the aerosol signal from the molecular channel. The ATR is defined as:

$$
A T R=\frac{P_{A}}{P_{M}}
$$

where $\mathrm{P}_{\mathrm{A}}$ is the Mie signal measured by the aerosol channel, and $\mathrm{P}_{\mathrm{M}}$ is the Mie signal measured by the molecular channel. Since the best possible performance is achieved when $\mathrm{P}_{\mathrm{M}}=0$, an infinite ATR would represent perfect performance. This is not achievable in practice, and an ATR greater than 10 is deemed acceptable to provide adequate measurements. For a spaceflight version of the instrument, and future aircraft iterations, it is desired to achieve an ATR greater than 30 , with a goal of greater than 50 .

In order to achieve high ATR values, two considerations must be taken into account: the optical path difference of the interferometer, and wavefront errors induced by the geometry and materials of the system. Both of these parameters influence the measured values of $\mathrm{P}_{\mathrm{A}}$ and $\mathrm{P}_{\mathrm{M}}$. In a perfect system, no wavefront errors are present, and the output of the interferometer in each channel is a plane-wave without tilt (Figure 3a). In this case, the optical path difference alone determines the ATR value, and the air arm pressure can be adjusted to achieve fully destructive interference at the molecular channel $\left(\mathrm{P}_{\mathrm{M}}=0\right)$. In practice, it is very difficult to build an interferometer that does not induce wavefront errors of some kind into the output.

Wavefront error can appear in the system in a number of forms. Tilt error (Figure 3b) is the most pronounced after assembly. Tilt error occurs when the optical path difference varies primarily in one direction across the aperture, and is generally due to manufacturing tolerance limits. OPD variation greater than one wavelength will appear as a fringe pattern. Residual errors are other random errors in the wavefront that are due to material non-uniformity (Figure 3c) and polishing errors. After initial measurements are taken with the interferometer, the measured wavefront errors at one particular temperature can be almost fully removed by a fluid jet polishing technique developed by LightMachinery Inc. After this process, the interferometer output will be very close to a plane wave if the system temperature is maintained at the polishing temperature. 


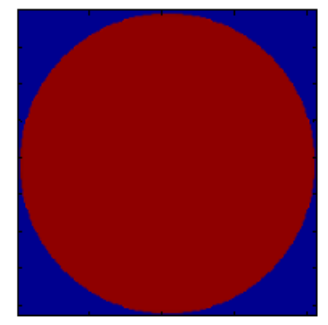

a.) Plane-wave, no error, goal after polishing

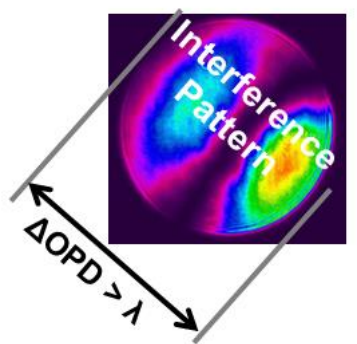

b.) Tilt, OPD varies in one direction across aperture

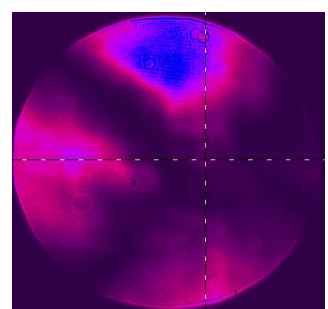

c.) Residual, random variation of OPD after removing tilt.

Figure 3. Examples of wavefront error viewed by detector, irradiance.

If the operating temperature cannot be maintained at the same temperature that polishing measurements were taken, then wavefront errors can again appear after the final polishing process. If the temperature varies enough, coefficient of thermal expansion (CTE) mismatch effects between the air arm spacer material and air arm mirror cause shape changes in the mirror. Additionally, temperature gradients in the glass arm that vary from the gradients that existed when the polishing measurements were taken cause wavefront errors due to refractive index changes across the glass. These shape and refractive index changes are visible in the interference output of the interferometer and can be characterized in terms of classical optical Seidel aberrations or in terms of Zernike polynomials.

The obvious solution to these issues is to maintain the interferometer at the same temperature condition at which the residuals were measured for the final polishing. This can be challenging on the aircraft since the ambient temperature is continuously changing during the flight. The aircraft design includes a thermal control housing that surrounds the interferometer, and helps to alleviate this changing environment by maintaining the interferometer at a slightly higher temperature than the maximum expected ambient temperature on the aircraft. However, it cannot perfectly maintain a uniform temperature condition in the system. Therefore, it would be desirable to achieve acceptable performance over a small temperature range to allow flexibility in the design.

\section{STOP MODEL DEVELOPMENT}

A detailed STOP model of the interferometer was developed using Comet to provide a design tool capable of predicting the performance of the system under realistic environmental conditions. The ultimate goal of the model is to optimize the design of the interferometer to ensure that adequate ATR is achieved over a specified operating temperature range. To perform this optimization, the model must have the flexibility to easily change the geometry of components, and easily change boundary conditions, while providing a way to link a large number of analysis tools, including structural, thermal, optical, and custom post-processing scripts. The following sub-sections describe the modeling approach in detail.

\subsection{Comet Modeling Methodology and STOP Process Development}

The Comet software package allows for the development of abstract analysis processes that are initially independent from geometry. This lets discipline-engineers work together to conceptually define the analyses needed to verify the design requirements in a single location. Once the process is defined, a CAD model representing the system to be studied is "tagged" in a way that parts, materials, and boundary conditions will be applied appropriately by the developed process. The CAD is then imported into the process, and all required analyses are performed sequentially. This is especially useful for systems requiring integrated analyses between disciplines, as it eliminates the need for individual engineers to perform independent analyses, and then pass their results to the next engineer in the analysis sequence. Once this process is developed, any of the discipline engineers can modify parameters of the system, run the entire analysis process, and determine how their design decisions affect the overall performance of the system.

Using this methodology, an integrated STOP process for the interferometer was developed in Comet, as shown in Figure 4. The major portions of the process are outlined in the red dashed boxes, and labeled appropriately. Each object in the process represents a particular task or analysis to be performed, and the black lines represent data exchanges between 
tasks. Tasks in the process are run sequentially based on the required data exchanges for each task; therefore a task cannot run until all "upstream" tasks that provide it information have been completed.

In the "CAD input and Meshing" section, the CAD model is imported, and two separate finite element meshes are created, one for the thermal model, and one for the structural model. Both of these meshes are passed to the "Thermal Analysis" section, where the system temperatures are predicted using Thermal Desktop ${ }^{\circledR}$, and these results are then mapped to the structural mesh with the same tool. In the "Structural Analysis" section, the mapped temperatures are added to the structural loads definition, and the structural mesh is sent to the structural analysis task, which is performed by NASTRANTM. The "Air and Glass Refractive Index Calculation" section utilizes MATLAB ${ }^{\circledR}$ to calculate the refractive index of air in the air arm, based on the predicted temperature from the thermal model. It also calculates a reference value for the refractive index of the glass, and the thermal coefficient $(\mathrm{dn} / \mathrm{dT})$ of the glass based on the manufacturer-provided dispersion equations. The "Map Therm/Struc to Optics" section uses SigFit ${ }^{\mathrm{TM}}$ to calculate the Zernike representations of the wavefronts in each arm from the predicted deformations and predicted thermal gradients on the optical components. These Zernike files are then passed to the "Optical Analysis" section, which utilizes CODE $\mathrm{V}^{\mathrm{TM}}$ to calculate the optical path difference of the wavefront in the form of phase matrices for the solid arm and for the air arm over the interferometer exit pupil. In addition, CODE V ${ }^{\mathrm{TM}}$ calculates the optical path length of the center-ray for each arm. The phase matrices and optical path lengths are then passed to the "Post Processing" section, where a custom MATLAB $^{\circledR}$ program calculates the interferogram, converts it to irradiance, and determines the ATR. The MATLAB ${ }^{\circledR}$ code then prints all the appropriate images and results of the analysis to a Power Point file. The details of each individual model and the associated boundary conditions are presented in the next sub-sections.

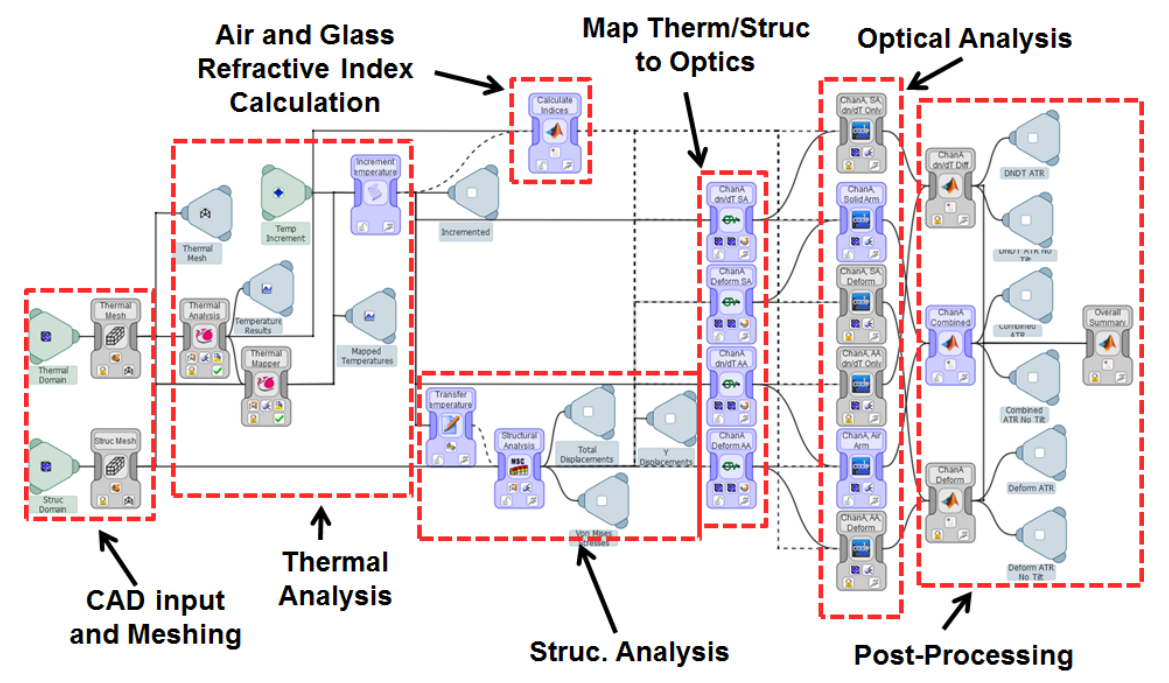

Figure 4. Interferometer STOP analysis process developed in Comet.

\subsection{Meshing}

The meshing section of the process takes the tagged interferometer CAD geometry, and creates independent finite element meshes for the thermal analysis and structural analysis. The thermal model does not need as fine a mesh as the structural model, and using two different mesh sizes saves computational time in the thermal analysis task. The element types, element sizes, and many other meshing parameters can be specified in the individual meshing tasks. An example of the meshes (2D view of a 3D mesh) is shown in Figure 5. 


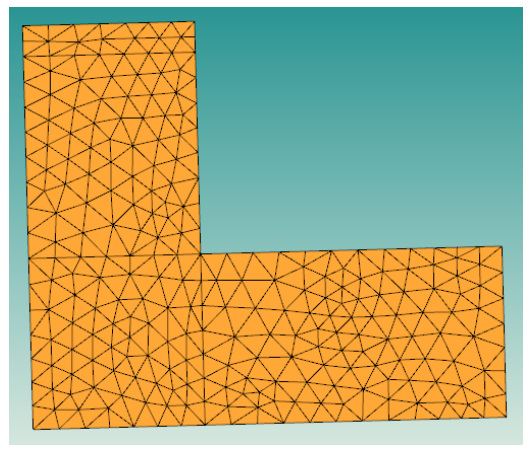

a.) Thermal Mesh

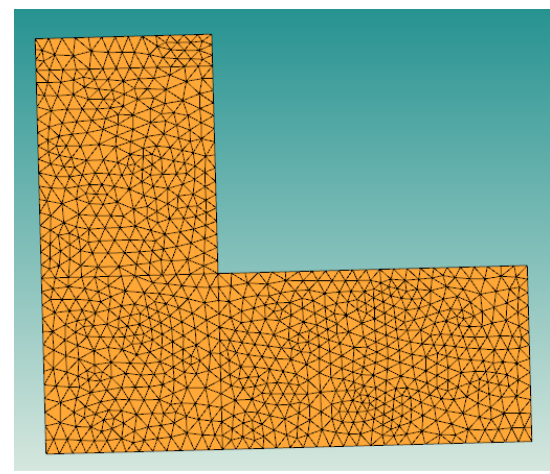

b.) Structural Mesh

Figure 5. Example mesh representations of interferometer (2D view of a 3D mesh).

\subsection{Thermal Analysis}

In the thermal analysis section of the process, two separate Thermal Desktop ${ }^{\circledR}$ tasks are used: one to perform the thermal analysis, and the other to map the temperature results from the thermal mesh to the structural mesh. The thermal analysis task utilizes a Comet environment, which defines the loads and boundary conditions for the model. The environment is designed to handle a number of different scenarios, which are parameterized through variables in the software. It can simulate a uniform temperature change of the interferometer, or simulate realistic boundary conditions imparted to the interferometer from the thermal control housing around the system. For the aircraft version of the model, the thermal control housing is pre-defined in a Thermal Desktop ${ }^{\circledR}$ template model, which is used as the starting point of the thermal analysis task. The thermal analysis task imports the interferometer mesh from Comet into the template model, and updates the boundary conditions specified for the particular analysis being performed through the environment definition. If the housing is being used, convection and radiation can be simulated between the housing and the interferometer. Conduction losses through the mounting feet are simulated through thermal contactors in the model. An example of the template model thermal control housing, and some thermal results on the interferometer are shown in Figure 6.

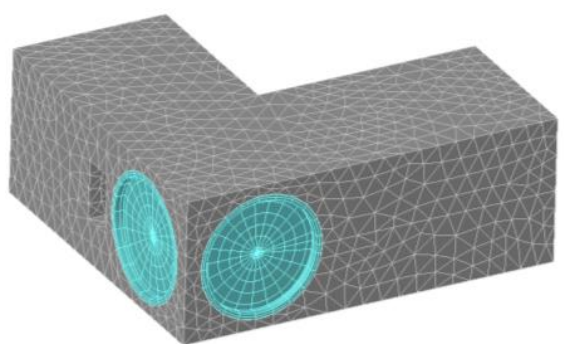

a.) Thermal housing template

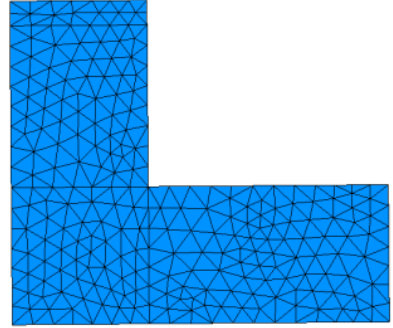

b.) Uniform interferometer temp

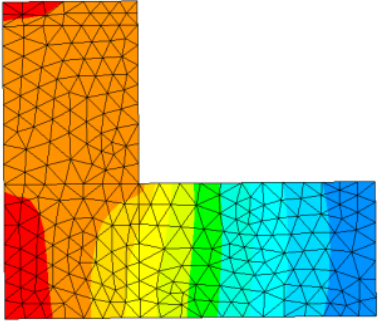

c.) Interferometer Temperature gradient

Figure 6. Example thermal analyses.

\subsection{Structural Analysis}

After the thermal analysis is performed, the temperature predictions on the interferometer are mapped to the structural mesh. This mapped temperature field is applied as a load to the structural environment set on the MSC NASTRANTM analysis task. The environment also defines the initial temperature of the system, and the appropriate constraints at the location of the mounting feet. The task performs a static structural analysis (NASTRAN ${ }^{\mathrm{TM}} 101$ solution) to predict the deformations of interferometer when subjected to the applied temperature field calculated by the thermal model. An example of the structural deformation results are shown in Figure 7. 


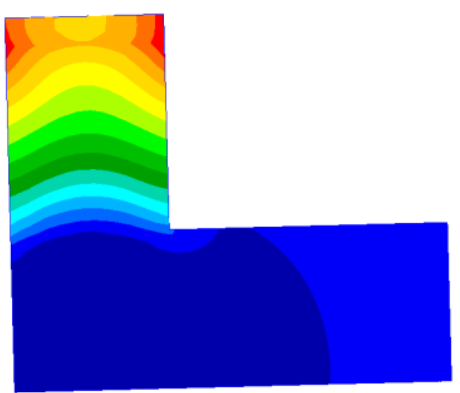

Figure 7. Example deformation results.

\subsection{Refractive Index Calculations and Mapping Thermal and Structural Analysis to Optics}

In the Comet model, variables are defined for the pressure in the air arm and the wavelength of the input beam. A MATLAB $^{\circledR}$ task uses these values, along with the average temperature of the air arm found in the thermal model, to calculate the refractive index of air using an equation from reference [6].

In addition, the same MATLAB ${ }^{\circledR}$ task calculates the reference index and the temperature coefficient (dn/dT) of the glass based on the dispersion equations provided by the manufacturer and the wavelength entered in the model. The glass reference index and $\mathrm{dn} / \mathrm{dT}$ are entered into the two thermally induced refractive index change SigFit ${ }^{\mathrm{TM}}$ tasks, one for each arm. These two tasks use the glass index and dn/dT, along with the mapped thermal analysis results on the structural mesh and the optical sequence files, to calculate the wavefront disturbance through the glass components due to changes in refractive index with temperature. The air in the air arm is not explicitly modeled in the finite element model, and therefore SigFit ${ }^{\mathrm{TM}}$ does not require its index or dn/dT. Two additional optical surface deformation SigFit ${ }^{\mathrm{TM}}$ tasks calculate the optical disturbances due to the deformations of the interferometer as calculated in the NASTRANTM analysis. The disturbances from all four tasks in the form of Zernike interferogram files are then passed to the optical analysis tasks.

\subsection{Optical Analysis}

The optical analysis is separated into two CODE VTM tasks, one for the air arm, and one for the solid arm. The optical ray traces for each arm are shown in Figure 8; the slight entry angle of the actual system is ignored in the analysis. The beam diameter is set to $30 \mathrm{~mm}$ to match the lab setup for testing the interferometer. For each arm, the respective deformation and thermally induced refractive index change Zernike files calculated with SigFit ${ }^{\mathrm{TM}}$ are attached to the respective surfaces in the optical model. The calculated index of air from the MATLAB ${ }^{\circledR}$ task is also applied to the air arm optical model. The Beam Synthesis Propagation (BSP) option is used in each optical model to calculate the phase in each arm at the detector, taking into account the disturbances calculated in SigFit ${ }^{\text {TM }}$. In addition, an Optical Path length (OP) command is used to determine the optical path length from the entry face of the beam splitter to the exit face of the beam splitter. This information is used in the post-processing section of the STOP process to calculate the correct interferogram.

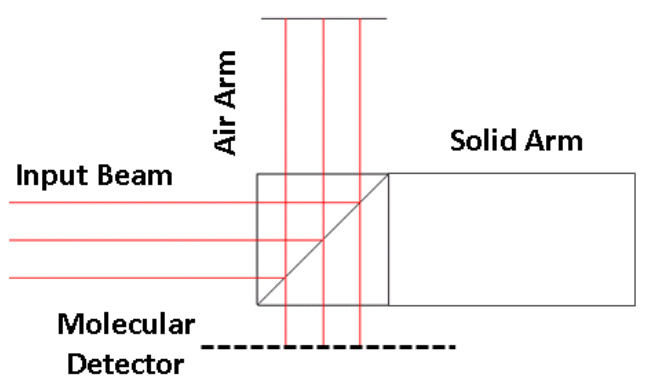

a.) Air arm optical model

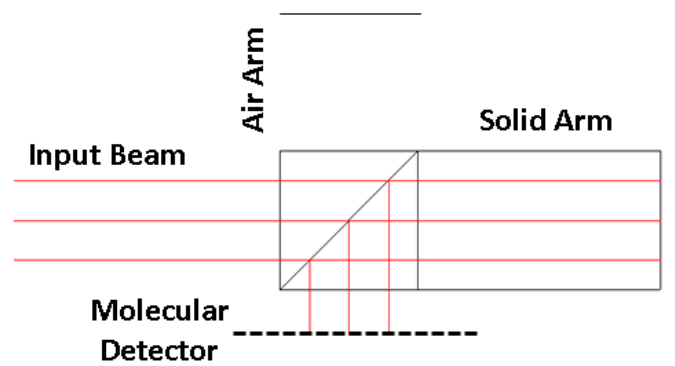

b.) Solid arm optical model

Figure 8. Optical models for each arm of the interferometer. 


\subsection{Post-Processing}

A final MATLAB ${ }^{\circledR}$ task is used to perform a number of functions. The most important of these are the calculation of the interferogram, the calculation of the ATR, and writing the results of the full process to a PowerPoint summary file. The two detector phase matrices calculated by the optical BSP routine are imported into MATLAB ${ }^{\circledR}$, and one is subtracted from the other to simulate the interference between the two arms. Since the phase matrix calculated by BSP is relative to the phase of the center ray, no information about optical path difference between the arms is present in this calculation. The optical path lengths calculated for the center ray in each arm are used to determine the phase shift between the arms by the following formula:

$$
\Delta \Phi=R E M A I N D E R\left(\frac{O P L_{S A}-O P L_{A A}}{\lambda}\right) * 2 * \pi
$$

where $\Delta \Phi$ is the phase difference between the arms, $\mathrm{OPL}_{\mathrm{SA}}$ and $\mathrm{OPL}_{\mathrm{AA}}$ are the optical path lengths (in $\mathrm{nm}$ ) of the solid arm and air arm respectively, and $\lambda$ is the wavelength in $\mathrm{nm}$. This value is then added to all values of the calculated interferogram from the previous step.

In order to calculate ATR, the phase at the single detector is converted to amplitude to simulate the irradiance measured by the detector using the following formula:

$$
P_{M}=\frac{1}{2}+\frac{1}{2} * \cos \left(\Phi_{M}\right)
$$

where $\mathrm{P}_{\mathrm{M}}$ is the irradiance matrix at the molecular channel detector, and $\Phi_{\mathrm{M}}$ is the phase matrix of the interferogram at the molecular detector. Theoretically, the phase at the aerosol channel detector is shifted $180^{\circ}$ from the phase at the molecular channel detector, so the irradiance at the aerosol channel can be approximated by

$$
P_{A}=\frac{1}{2}+\frac{1}{2} * \cos \left(\Phi_{M}+\pi\right)
$$

and the ATR can be calculated as

$$
\operatorname{ATR}=\frac{\operatorname{sum}\left(P_{A}\right)}{\operatorname{sum}\left(P_{M}\right)}
$$

where the elements of the irradiance matrix are summed over the detector aperture. It should be noted that in the actual instrument, the ATR is a derived value from measurements on each detector. The STOP model includes only one output of the interferometer, and uses the theoretical leakage of the Mie signal into the other output to calculate ATR.

In addition to the calculations shown above, the MATLAB ${ }^{\circledR}$ code also performs a numerical tilt removal from the interferogram phase. The purpose of this tilt removal is to allow the calculation of ATR if the interferometer could be built without manufacturing tolerance errors. This "tilt-free" ATR is a useful parameter, as it approximates what can be achieved after fluid jet polishing the mirror. However, it is not a perfect simulation of this process since it does not remove other sources of wavefront error.

At the end of the script, the optics results, including images of the detector phase and irradiance at each step of the calculation, are written to a power point file using the free MATLAB ${ }^{\circledR}$ script "saveppt.m". output slides are shown in Figure 9. 


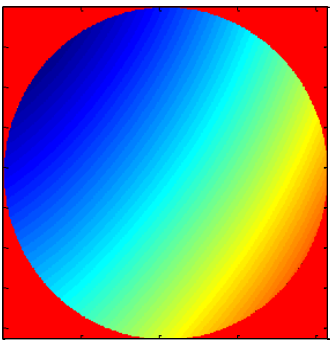

a.) Phase image

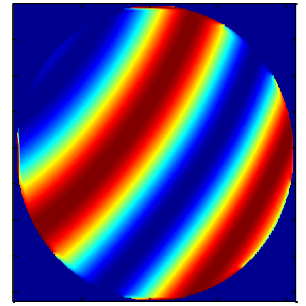

b.) Irradiance (amplitude) image

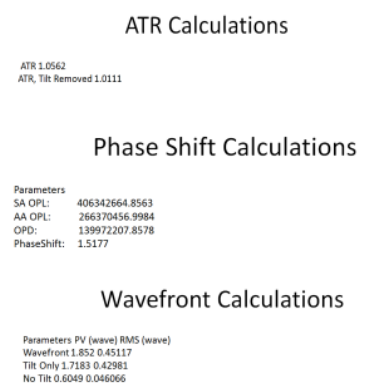

c.) ATR and other calculation slides

Figure 9. Sample results automatically written to PowerPoint by the post-processing task.

\section{STOP ANALYSIS VERIFICATION AND RESULTS}

After the analysis process was completed, a number of verification analyses were performed to ensure the model yields expected results for known inputs. These analyses included checks of both thermal and non-thermal effects. Once completed, the model was used to simulate a transient temperature ramp of the interferometer during a lab test, as well as to compare the effects of increasing temperature on ATR. The model was then used to perform a number of trade studies to improve the performance of the system.

\subsection{Non-Thermal Verification Analyses}

In each of the tests described in this section, the temperature of the interferometer was kept at the assembly temperature $\left(20^{\circ} \mathrm{C}\right)$ so no expansion due to CTE was present. In each test case, a parameter was varied by an amount known to cause a change in interferometer optical path difference of one wave, or one free spectral range (FSR). The three analyses performed are as follows:

1. Air arm mirror displacement of $1 / 2$ wavelength should yield a 1 FSR change $(\triangle \mathrm{OPD}=1 * \lambda)$

2. Wavelength change of $2 \mathrm{GHz}(0.84 \mathrm{pm})$ should yield a 1 FSR change $(\Delta \Phi=2 \pi)$

3. Air arm pressure change of 10.87 mbar should yield a 1 FSR change $(\triangle \mathrm{OPD}=1 * \lambda)$

The results of these STOP analyses are shown in Figure 10, and each study yields the expected one wave change in OPD, or phase shift by $2 \pi$, providing confidence that the model is representative of the physics of the system. Each plot required 5 STOP analyses to be performed, and was automated by using a Comet parameter study. The wavelength, pressure, and air arm displacement are defined as variables in the model, and the parameter study increments the variable and reruns the analysis automatically. The 15 STOP analyses depicted in the plots were performed in less than 4 hours on a single laptop with an 8 core $2.2 \mathrm{GHz}$ processor, and $8 \mathrm{~GB}$ of memory. Performing this analysis manually would take significantly longer since each engineer would need to perform their analysis independently, and pass results to the next engineer in the analysis sequence.

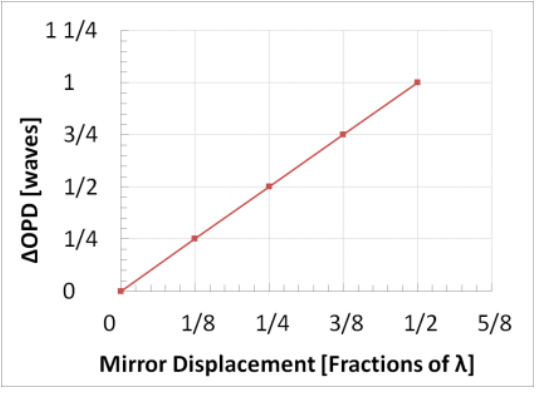

a.) 1/2 wavelength mirror displacement

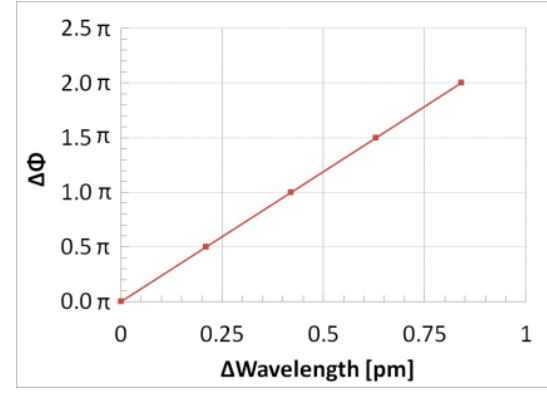

b.) $0.84 \mathrm{pm}$ wavelength change

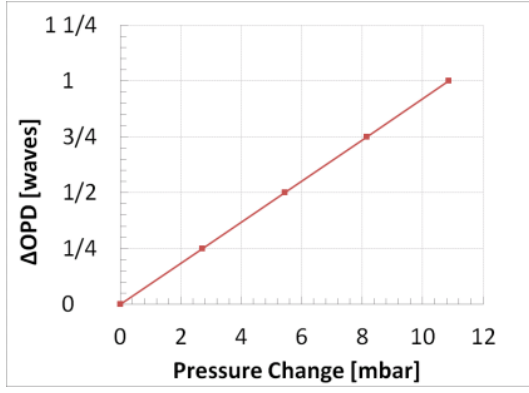

c.) 10.87 mbar pressure change

Figure 10. Results of non-thermal verification studies. 


\subsection{Thermal Verification Analyses and CTE Mismatch Effects}

The optical path difference of the interferometer can be calculated analytically for a uniform temperature change, under the assumption that CTE mismatch effects are negligible. It is simply the difference of the optical path lengths of the two arms, taking the initial geometry and material properties into account:

$$
O P D=O P L_{S A}-O P L_{A A}=n_{S A}(T, \lambda) \cdot L_{S A} \cdot\left(1+C T E_{S A} \cdot \Delta T\right)-n_{A A}(T, \lambda, P) \cdot L_{A A} \cdot\left(1+C T E_{A A} \cdot \Delta T\right)
$$

where $\mathrm{n}_{\mathrm{SA}}, \mathrm{L}_{\mathrm{SA}}, \mathrm{CTE}_{\mathrm{SA}}$ are the refractive index, length, and $\mathrm{CTE}$ of the solid arm respectively, and $\mathrm{n}_{\mathrm{AA}}, \mathrm{L}_{\mathrm{AA}}, \mathrm{CTE}_{\mathrm{AA}}$ are the corresponding variables for the air arm.

Figure 11a shows the difference between the predicted OPD using the equation above, and the STOP model for the asbuilt interferometer geometry and material properties. At the reference temperature (assembly temperature) of $20^{\circ} \mathrm{C}$, there is no difference in the predictions of the two methods, which provides confidence that the STOP model is correctly simulating the OPD in the un-deformed state. At a temperature of $30^{\circ} \mathrm{C}$, however, there are approximately 2.3 waves of difference between the model prediction and equation prediction. The air arm contains bonded interfaces of dissimilar materials, which causes CTE mismatch stresses to arise when the system is heated, and the difference in OPD of the model compared to the equation is due to this effect. This is verified in Figure 11b, where all parts in the STOP model are given the same value for CTE. In this case, CTE mismatch effects are eliminated, and two calculation methods match within 0.01 waves at both temperatures.

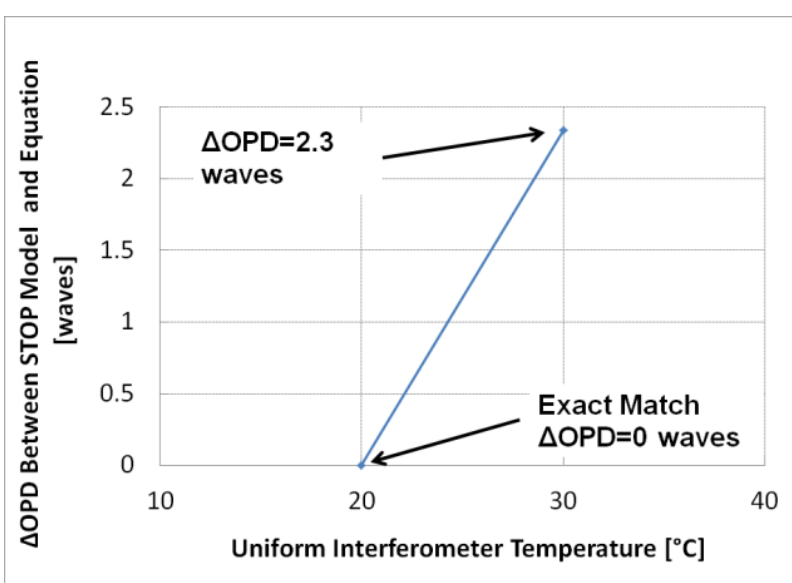

a.) $\triangle O P D$ between STOP prediction and OPD equation for the as-built geometry and material properties

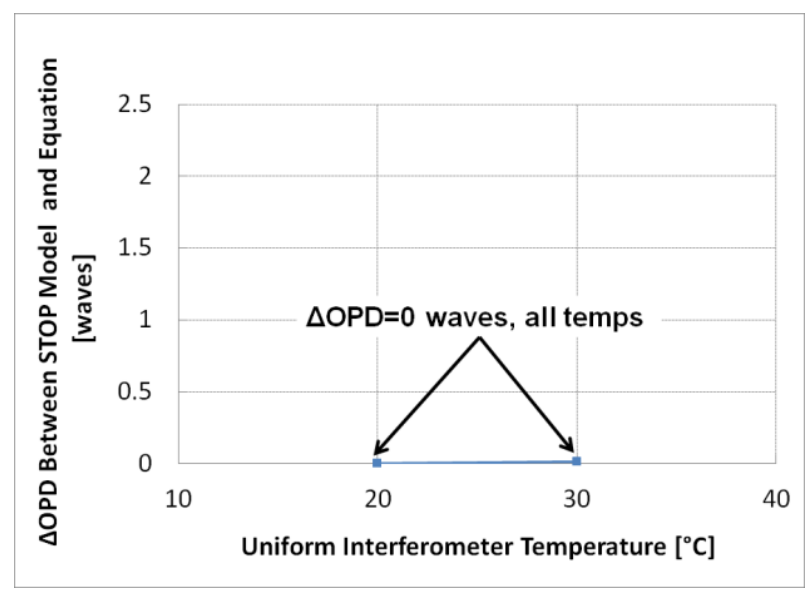

b.) $\triangle$ OPD between STOP prediction and OPD equation for the as-built geometry and all part CTEs assumed equivalent to eliminate mismatch effects

Figure 11. STOP OPD predictions compared to equation, showing CTE mismatch can have an effect on the results.

\subsection{Comparison to Lab Testing}

The interferometer and thermal control housing was setup in a lab environment in order to measure the wavefront error in the system for future polishing. A number of additional tests were conducted to verify the performance of the system, including testing at steady state temperatures and testing during a transient temperature increase of the housing. The lab setup consisted of a $355 \mathrm{~nm}$ tunable single-frequency laser, collimated to a $30 \mathrm{~mm}$ beam before entering the interferometer, and a CCD detector to measure the spatial properties of the output beams. All testing presented was done before fluid jet correction of the wavefront error, and all CCD images contain about 1.5 waves of tilt (due to initial manufacturing tolerances), and some residual error. In order to simulate the tilt error present in the interferometer, the air arm mirror in the STOP model was also tilted to produce a fringe pattern that closely matched the recorded images in the lab.

For the transient temperature test, the thermal control housing surrounding the interferometer was quickly heated from approximately $20^{\circ} \mathrm{C}$ to $35^{\circ} \mathrm{C}$. The temperature of the control housing was recorded by multiple RTDs during the test, and CCD images were recorded every 3 seconds. A transient thermal analysis was performed using the STOP model to simulate the test conditions. The measured housing temperature and measured mounting surface temperature were used 
as boundary conditions in the model, and the analysis was performed for 120 minutes. Results of the thermal analysis are shown in Figure 12. The thermal housing curve represents the measured value of the thermal housing during testing. Predicted results for the center of the air arm mirror, the center of the solid arm, and the center of the beam splitter cube are also displayed.

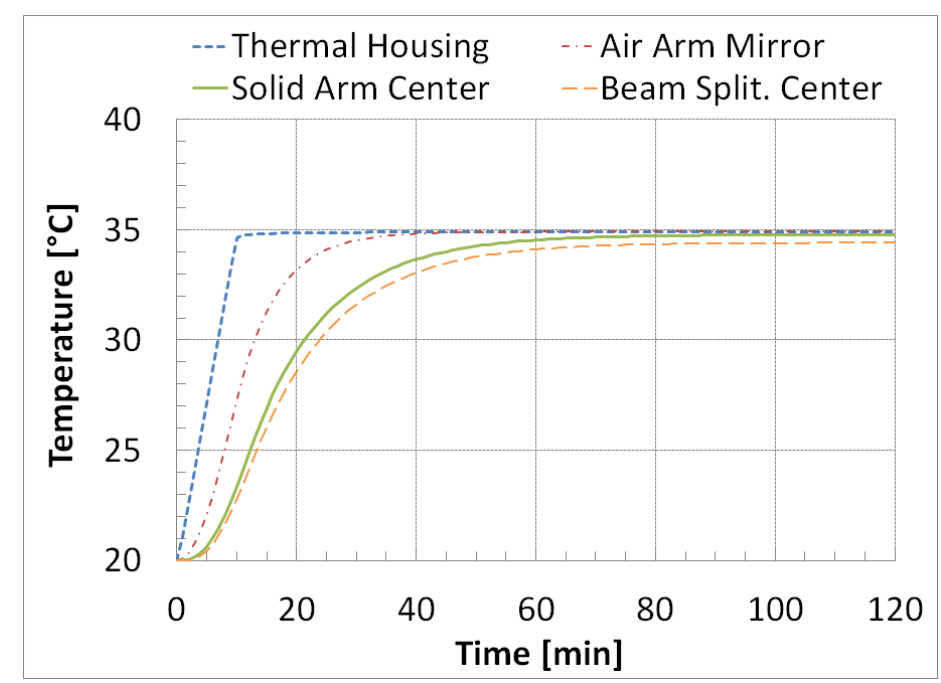

Figure 12. STOP transient thermal analysis using measured housing temperature as boundary condition.

The transient thermal analysis saved temperature predictions for the interferometer every 1 minute, yielding a total of 120 independent temperature fields for the interferometer. A Comet parameter study was then used to automatically perform a STOP analysis for every temperature field during the transient so that the predicted detector image could be compared to the CCD images taken during the lab test. This comparison is shown for key time points in Figure 13. At the beginning of the test, the interferometer is uniform in temperature, and the tilt in the STOP model was added to simulate the tilt in the as-built system. Note the difference in appearance in the STOP model fringes and the labmeasured fringes in figure 13a, 13c, and 13d. The reason for this is that the STOP model uses a uniform illumination of the interferometer, while the laboratory case uses a Gaussian laser beam to illuminate the interferometer. Thus the laboratory images decrease in intensity near the ends of the fringes. At time $=10$ minutes, a radial temperature gradient has formed in the interferometer, and radial fringes appear in both lab results and the STOP model. At approximately this time, the maximum number of fringes is seen in both cases. By 90 minutes, the interferometer temperature is nearly uniform, and very little fringe movement is seen for the remainder of the test. While the transient behavior is not exactly captured by the STOP analysis, it demonstrates the ability of the model to capture the basic physics of the test, and provides confidence in using it as a tool to inform design decisions for future iterations of the interferometer. The 120 STOP analyses performed for this simulation were automatically run overnight on the same laptop computer previously described. The irradiance images were printed to a single PowerPoint file during the run to enable a movie to be generated easily from the analysis. If performed manually, this would require 120 thermal-structural mappings, 120 structural analyses for each mapped temperature, 480 optics mapping analyses (thermal and deformations, one for each arm), 240 optical analyses, and 120 post processing analyses. This would be performed sequentially by three engineers, and would take much longer than a single overnight run. 
Detector Irradiance:

STOP Prediction
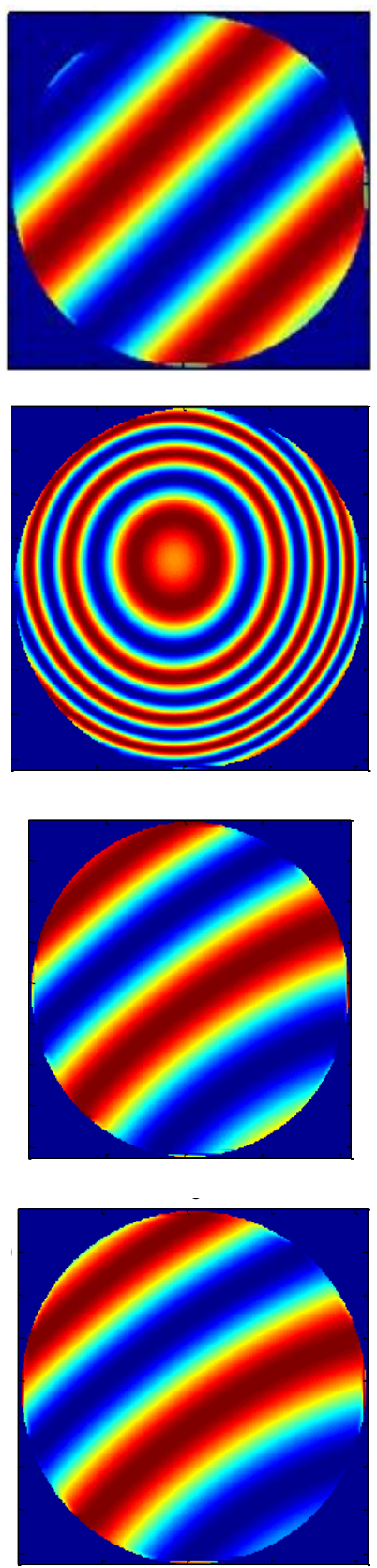

Detector Irradiance:

CCD Image From Lab Test
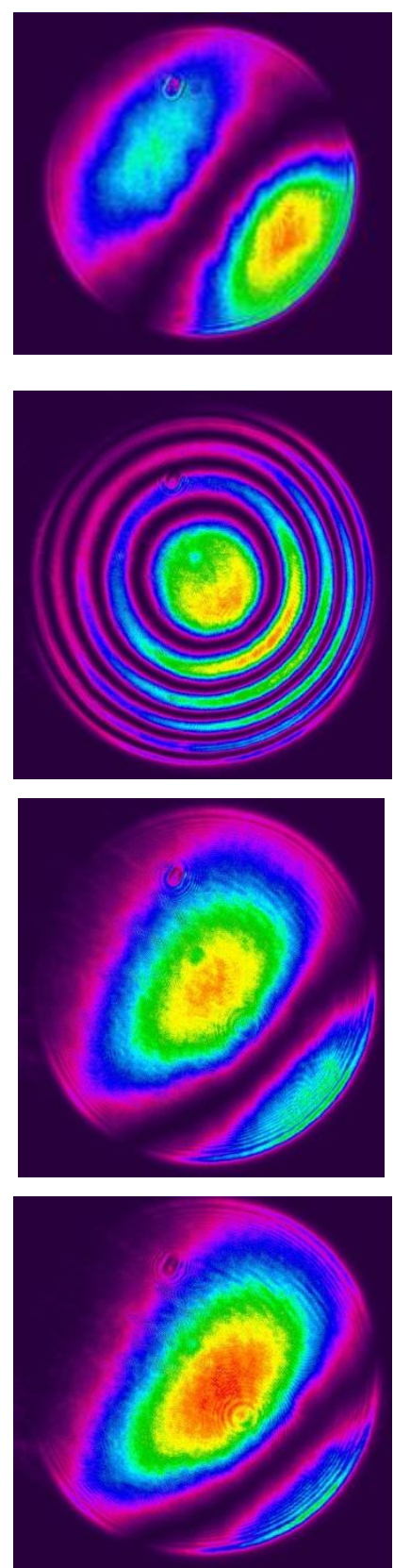

Time $=120 \mathrm{~min}$, end of test, very small change in

fringe position from $90 \mathrm{~min}$

Time $=90 \mathrm{~min}$, interferometer temperature nearly uniform, fringe motion nearly stopped

Time = 10 min, max number of fringes, radial temp gradient in solid arm and beam splitter causes radial fringes to appear

Figure 13. Comparison of detector images between lab test and STOP model for a transient temperature ramp. 
In order to determine how the ATR is affected by temperature changes, a number of lab tests were conducted with the interferometer at steady temperature values. $A$ MATLAB $^{\circledR}$ routine similar to the one used in the STOP model was used to post-process the lab fringe images, and calculate the tilt-removed ATR at each temperature value. As discussed previously, tilt-removed ATR is calculated by numerically removing the tilt from the irradiance images before calculating ATR. It cannot remove other aberrations from the image; however, it gives a good indication of the ATR that can be expected after polishing since tilt is usually the major form of wavefront error in the system. A comparison of tilt-removed ATR between the lab tests and the STOP model is shown in Figure 14. Both curves show that the ATR degrades as the temperature of the interferometer deviates from the temperature at which it was assembled. Based on STOP model results, this degradation appears to be caused by warping of the air arm mirror due to CTE mismatch effects between the mirror and air arm spacer. This warping can be seen by comparing the first and last STOP irradiance image in Figure 13. This curvature is also seen in the lab test results, but is not as clear in the format of the images presented. The STOP model predicts higher ATR values overall than the lab testing because there are no aberrations in the system other than the tilt that was added to match the lab images. The ATR at $20^{\circ} \mathrm{C}$ would be infinite; however, there are some numerical limitations in the tilt removal calculation. The fact that the model predicts a similar degradation to the lab testing is the key point. Design studies can be performed to reduce this degradation with some confidence that it will accurately match a built system.

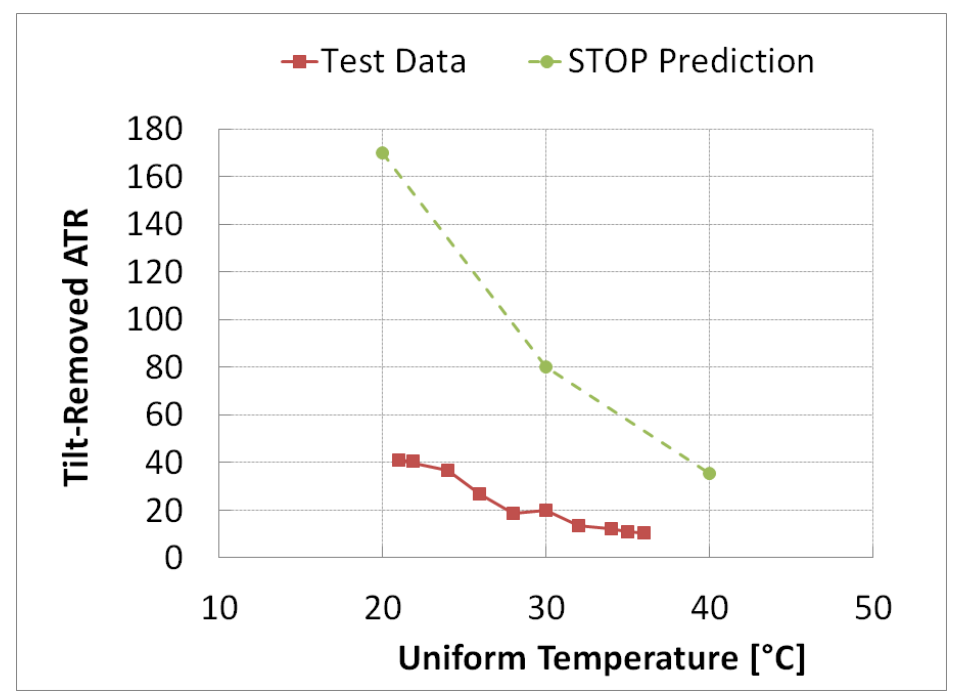

Figure 14. Tilt-removed ATR vs. temperature comparison between STOP model and lab testing

\subsection{Design Trade Studies}

Based on information from the lab testing, and results from the STOP model, a few items were identified that could be changed to improve the performance of the system. One of these items was the mirror warping discussed in the previous section, which appear to be related to CTE mismatch effects in the air arm assembly. A trade study consisting of 11 different geometric or material configurations was performed. Some of these were not simply dimensional changes, but involved adding parts or modifying parts in the CAD model. For each configuration, three STOP analyses were performed at uniform temperatures of $20^{\circ} \mathrm{C}, 30^{\circ} \mathrm{C}$, and $40^{\circ} \mathrm{C}$. Taking the simplicity of the design option into account, the best performance improvement could be obtained by doubling the thickness of the mirror, or adding a CTE "compensator" part to the backside of the mirror. The results of these two studies are shown in Figure 15 for the $40^{\circ} \mathrm{C}$ case, including the comparison to the original design at the same temperature. A significant improvement in the fringe warping can be obtained using one of these methods, and should improve the maximum ATR achievable at temperatures away from the assembly temperature. The 33 STOP analyses, including CAD modifications and documentation of results, were performed within 2 days using Comet, and the previously described laptop. 


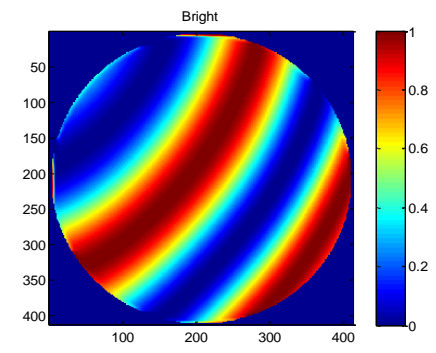

a.) Original design, $40^{\circ} \mathrm{C}$

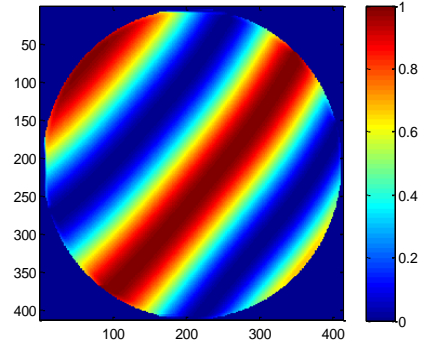

b.) Double-thick air arm mirror, $40^{\circ} \mathrm{C}$

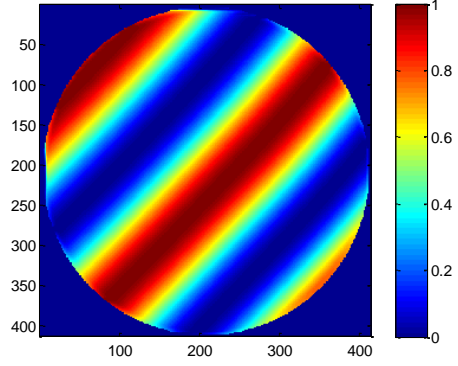

a.) CTE compensator, $40^{\circ} \mathrm{C}$

Figure 15. Comparison of design changes intended to reduce mirror warping at elevated temperatures.

\section{CONCLUSIONS}

An integrated STOP model of the HSRL interferometer was developed by defining a reusable, automated analysis process with Comet. The model predicts the results of theoretical cases with known results exactly. It shows good agreement with lab test results, and this builds confidence in the ability to use the model to inform design decisions. Trade studies using the model have demonstrated modifications that can improve the performance of the interferometer. Additional trade studies and lab tests will be performed, and an improved version of the interferometer will be built in 2015 based on the results of the studies. This version will undergo a spaceflight qualification test campaign to mature the technology for future space missions.

A powerful benefit of building an analytical model with an integrated tool like Comet is the ability to perform trade studies very rapidly, as demonstrated by the transient simulation and mirror warping analyses described. While there is a significant amount of time required upfront to develop the process, the analytical speed benefits appear to be worth the initial investment. In addition, the upfront time is likely to be no more than would be required if independent thermal, structural, and CAD models were developed in a traditional fashion. Most variables in the model can be parameterized, which enables automated trade studies on boundary conditions, loads, material properties, and geometric dimensions. In addition, parts can be completely modified in the CAD model, and then run through the analysis process relatively quickly as compared to the traditional method of passing information between analysts to perform manual model updates. Significant reductions in analysis design cycle durations for systems requiring integrated analyses could be realized as the software continues to evolve.

\section{ACKNOWLEDGEMENTS}

The authors would like to thank Jean-Claude Mahuet and the support team at Comet Solutions, Greg Michels of Sigmadyne, and Mary Kate Crawford of Synopsys, for their excellent support with software questions, bug fixes, and improvement requests. The team would also like to thank our branch heads and senior leadership in the Langley Engineering Directorate; this effort would not have been possible without their continued support.

\section{REFERENCES}

[1] Hair, J. W., Hostetler, C. A., Cook, A. L., Harper, D. B., Ferrare, R. A., Mack, T. L., Welch, W., Izquierdo, L. R., and Hovis, F. E.: Airborne High Spectral Resolution Lidar for profiling aerosol optical properties, Appl Optics, 47, 6734-6752, 10.1364/AO.47.006734, 2008.

[2] Burton, S. P., R. A. Ferrare, C. A. Hostetler, J. W. Hair, R. R. Rogers, M. D. Obland, C. F. Butler, A. L. Cook, D. B. Harper, and K. D. Froyd (2012), Aerosol Classification of Airborne High Spectral Resolution Lidar Measurements - Methodology and Examples, Atmospheric Measurement Techniques, 5(1), 73-98, doi: 10.5194/amt-5-73-2012. 
[3] S. T. Shipley, D. H. Tracy, E. W. Eloranta, J. T. Tauger, J. T. Sroga, F. L. Roesler, and J. A. Weinman, "High spectral resolution lidar to measure optical scattering properties of atmospheric aerosols. 1: Theory and instrumentation, Appl. Opt. 22, 3716-3724 (1983).

[4] P. Piironen and E.W. Eloranta, "Demonstration of a high-spectral- resolution lidar based on an iodine absorption filter," Opt. Lett. 19, 234-236 (1994).

[5] Comet Solutions ${ }^{\circledR}$, http://cometsolutions.com/

[6] Schott Technical Information, TIE-19. http://www.us.schott.com/advanced_optics/english/knowledgecenter/technical-articles-and-tools/tie.html

[7] MATLAB $^{\circledast}$ Central, saveppt.m. http://www.mathworks.com/matlabcentral/fileexchange/340-saveppt 